\title{
Rhein lysinate inhibits cell growth by modulating various mitogen-activated protein kinases in cervical cancer cells
}

\author{
YONG-ZHAN ZHEN ${ }^{1 *}$, YA-JUN LIN ${ }^{2 *}, J_{U N-L I N G ~ G A O}{ }^{1}$, YU-FANG ZHAO ${ }^{1}$ and AI-JUN XU ${ }^{1}$ \\ ${ }^{1}$ North China Coal Medical University, Tangshan, Hebei 063000; ${ }^{2}$ The Key Laboratory of Geriatrics, \\ Beijing Hospital and Beijing Institute of Geriatrics, Ministry of Health, Beijing 100730, P.R. China
}

Received August 3, 2010; Accepted October 20, 2010

DOI: $10.3892 / \mathrm{ol} .2010 .200$

\begin{abstract}
In previous studies, we found that rhein lysinate (RHL; the salt of rhein and lysine, easily dissolved in water) inhibited the growth of tumor cells in breast and ovarian cancer and hepatocellular carcinoma. This study aimed to investigate the effect of RHL on the growth of human cervical carcinoma HeLa cells and any underlying mechanisms. RHL inhibited the growth of HeLa cells in a dose- and time-dependent manner. It was also noted that RHL induced apoptosis in HeLa cells in a dose-dependent manner. Mechanistically, RHL triggered HeLa cell apoptosis by increasing the levels of cleaved poly ADP-ribose polymerase (PARP) and caspase-3/7. In addition, the activation of p38 mitogen-activated protein kinase (MAPK) and c-Jun NH2-terminal kinase (JNK) was a critical mediator in RHL-induced growth inhibition. Inhibition of the expression of p38 MAPK and JNK by pharmacological inhibitors reversed RHL-induced growth inhibition by decreasing the level of cleaved PARP and caspase-3/7. Phosphorylation of the extracellular signal-related kinase (ERK) was increased by RHL; conversely, the MEK inhibitor which inhibits ERK activity, synergistically enhanced RHL-induced growth inhibition in HeLa cells. The results showed that RHL inhibits Hela cell growth through the activation of p38 MAPK and JNK, and is a potential chemotherapeutic agent for cervical cancer.
\end{abstract}

\section{Introduction}

Cervical cancer is a major common cancer in women in developing countries, and it is the second most frequent cause of cancer-related death in women worldwide (1). Following

Correspondence to: Dr Ya-Jun Lin, The Key Laboratory of Geriatrics, Beijing Hospital and Beijing Institute of Geriatrics, Ministry of Health, Beijing 100730, P.R. China

E-mail: linyajun2000@126.com

*Contributed equally

Key words: rhein lysinate, cervical cancer, c-Jun NH2-terminal kinase, p38 mitogen-activated protein kinase, extracellular signalrelated kinase standard treatment, a high survival rate is noted in patients with cervical cancer. However, the issue of recurrence and subsequent resistance to chemoradiation therapy, as well as the mechanisms involved have yet to be elucidated.

Rhein, one of the major bioactive constituents of the rhizome of rhubarb $(2,3)$, inhibits the proliferation of various human cancer cells (4-8). Our previous study showed that rhein lysinate (RHL; the salt of rhein and lysine, easily dissolved in water) exhibits anti-cancer activity in breast and ovarian cancer and in hepatocellular carcinoma in vivo and in vitro $(9-11)$.

RHL affects various cell signaling pathways, and interest in its mechanisms has expanded to include a number of protein kinase pathways. The mitogen-activated protein kinase (MAPK) superfamily comprises a number of signaling pathways involved in growth regulation $(12,13)$. These pathways include extracellular signal-regulated kinase 1 (ERK1) and ERK2 (or p42 MAPK and p44 MAPK), c-Jun NH2-terminal kinases (JNKs) and p38 MAPK.

The potential role of RHL in the treatment of cervical cancer remains to be investigated and the mechanism by which RHL affects tumor growth remains to be determined. Therefore, the effects of RHL on cell growth, apoptosis and various MAPKs in cervical carcinoma HeLa cells were investigated.

\section{Materials and methods}

Chemicals and reagents. Rhein (98\%) was purchased from Nanjing Qingze Medicine Ltd. (Nanjing, Jiangsu, China). Lysine was purchased from Beijing Solarbio Science and Technology Co. (Beijing, China). RHL was created in our department (Patent No. 200810089025.8). 3-(4,5-dimethylthiazol-2-yl)-2,5-diphenyltetrazolium bromide (MTT) and dimethyl sulfoxide (DMSO) were obtained from Sigma Aldrich (Shanghai, China). The remaining chemicals were of standard analytical grade.

Cell culture. Human cervical carcinoma HeLa cell line was obtained from the Cell Center of the Institute of Basic Medical Sciences, Chinese Academy of Medical Sciences and Peking Union Medical College, China. The HeLa cells were cultured in Dulbecco's modified Eagle's medium (Gibco BRL, Grand Island, NY, USA) supplemented with $10 \%$ heat-inactivated fetal bovine serum (Sigma Chemical Co., St. Louis, MO, 
USA), $2 \mathrm{mM}$ glutamine, $100 \mathrm{U} / \mathrm{ml}$ penicillin and $100 \mu \mathrm{g} / \mathrm{ml}$ streptomycin at $37^{\circ} \mathrm{C}$ in a humidified atmosphere containing $5 \% \mathrm{CO}_{2}$.

Cell proliferation assay. The cell proliferation assay was examined with the MTT method, according to the manufacturer's instructions. Cells were seeded in 96-well plates (Costar, Cambridge, MA, USA) with 2,500 cells/well. Following overnight incubation, triplicate wells were treated with various concentrations of RHL for $48 \mathrm{~h}$. Then, $20 \mu \mathrm{l}$ MTT solutions $(5 \mathrm{mg} / \mathrm{ml}$ in PBS) were added to each well and incubated for $4 \mathrm{~h}$ at $37^{\circ} \mathrm{C}$. The MTT formazan was dissolved in $150 \mu \mathrm{l}$ DMSO and absorbance was measured using a Microplate Reader (Multiskan MK3; Thermo Labsystem, USA) at a wavelength of $570 \mathrm{~nm}$.

FITC-Annexin V/propidium iodide (PI) apoptosis assay. Cells were collected and resuspended in $200 \mu \mathrm{l}$ binding buffer. Then, $10 \mu 1$ FITC-labeled enhanced Annexin V (Baosai Biotechnology Ltd., Beijing, China) and 100 ng PI were added. Following incubation in the dark (15 min at room temperature or $30 \mathrm{~min}$ at $4^{\circ} \mathrm{C}$ ), the samples were diluted with $300 \mu \mathrm{l}$ binding buffer. Flow cytometry was carried out using a FACScan instrument (Becton-Dickinson) and the data were processed using WinMDI/PC-software.

Western blot analysis. Cells were harvested and washed with PBS solution. The whole cellular extracts were prepared by incubating cells on ice in lysis buffer containing $50 \mathrm{mM}$ Tris- $\mathrm{HCl} \mathrm{pH} \mathrm{7.5,}$ $150 \mathrm{mM} \mathrm{NaCl}, 2$ mM EDTA, 2 mM EGTA, $1 \mathrm{mM}$ dithiothreitol, $1 \%$ Nonidet P-40, $0.1 \%$ SDS, protease inhibitors (1 mM PMSF, $5 \mu \mathrm{g} / \mathrm{ml}$ aprotinin, $5 \mu \mathrm{g} / \mathrm{ml}$ leupeptin and $5 \mu \mathrm{g} / \mathrm{ml}$ pepstatin) and phosphatase inhibitors $(20 \mathrm{mM} \beta$-glycerophosphate, 50 $\mathrm{mM} \mathrm{NaF}$ and $1 \mathrm{mM} \mathrm{Na}_{3} \mathrm{VO}_{4}$ ). The cell lysates were cleared by centrifugation at $12,000 \mathrm{x}$ g for $12 \mathrm{~min}$. Protein concentrations were determined using the Bradford assay. Equal amounts of lysate $(40 \mu \mathrm{g})$ were resolved by SDS-PAGE and transferred to polyvinylidene difluoride membranes (Millipore Corp., Bedford, MA, USA). The membranes were blocked in TBST containing $5 \%$ non-fat skim milk at room temperature for $2 \mathrm{~h}$ and probed with primary antibodies overnight at $4^{\circ} \mathrm{C}$. Membranes were then blotted with an appropriate horseradish peroxidase-linked secondary antibody (Santa Cruz Biotechnology, Santa Cruz, CA, USA). Proteins were visualized using enhanced chemiluminescence Western blot detection reagents (Amersham Pharmacia Biotech, Inc., Piscataway, NJ, USA).

\section{Results}

Inhibition of proliferation by rhein lysinate in HeLa cells. The growth inhibitory effect of RHL on HeLa cells was examined using MTT assay. Cells were cultured for $48 \mathrm{~h}$, with or without different concentrations of RHL. A decreased cell proliferation following treatment with various concentrations of RHL is shown (Fig. 1A). The $\mathrm{IC}_{50}$ value of RHL for the HeLa cells was $\sim 80 \mu \mathrm{M}$. The cells were cultured with RHL $(50,75,100$ and $125 \mu \mathrm{M})$ for the indicated times and analyzed with the MTT assay (Fig. 1B). HeLa cells treated with the indicated dose of RHL showed decreased cell proliferation in a timedependent manner.

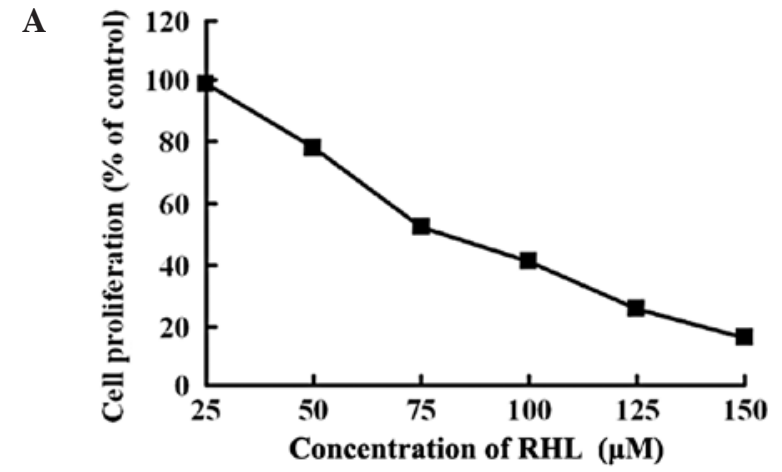

B

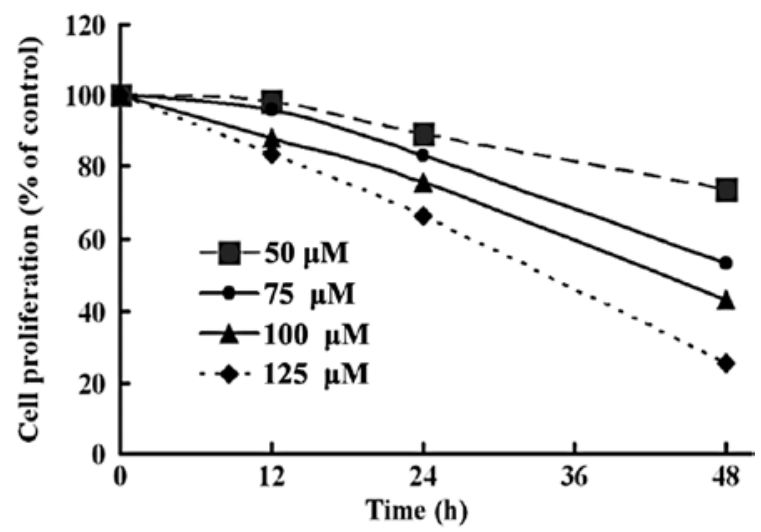

Figure 1. Effect of RHL on the cell proliferation of Hela cells. (A) The cells were treated with various concentrations of RHL at $37^{\circ} \mathrm{C}$ for $48 \mathrm{~h}$. The effects on cell proliferation were examined using MTT assay and the cell proliferation was calculated as the percentage of the control. (B) The cells were cultured with RHL $(50,75,100$ and $125 \mu \mathrm{M})$ for the indicated times and the effects on cell proliferation were also determined using MTT assay. Assays were performed in triplicate.

Apoptosis induction of rhein lysinate in HeLa cells. FITCAnnexin V/PI staining showed that RHL at $50 \mu \mathrm{M}$ induced early apoptosis in HeLa cells (Fig. 2A). The ratio of early apoptosis was significantly enhanced when cells were incubated with 75 or $100 \mu \mathrm{M}$ RHL for $48 \mathrm{~h}$. To determine whether RHL-induced apoptosis is mediated by the activation of caspase and/or PARP, HeLa cells were treated with RHL at various concentrations for $48 \mathrm{~h}$, RHL-induced caspase-3/7 and PARP cleavage in a dose-dependent manner (Fig. 2B). Taken together, these results strongly suggest that RHL triggers caspase-dependent apoptosis in HeLa cells.

Growth inhibition of rhein lysinate in HeLa cells was associated with the activation of various MAPKs. MAPKs are a family of proteins that transduce signals from the cell membrane to the nucleus in response to a wide range of stimuli and regulate vital biological functions, including gene expression, mitosis, differentiation, apoptosis, proliferation and motility $(12,13)$. Three major groups of MAPKs exist: JNK, p38 MAPK and ERK.

To investigate the molecular mechanisms underlying growth inhibition, the effect of RHL on the activities of MAPKs in HeLa cells was examined. As shown in Fig. 3, Western blot analysis revealed that RHL treatment increased the phosphorylation of JNK, p38 MAPK and ERK1/2 in a dose- and time-dependent manner in HeLa cells. These results 
A

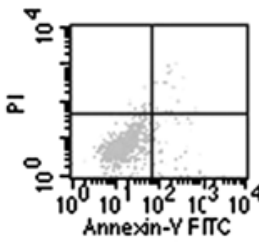

Control

$3.09 \%$

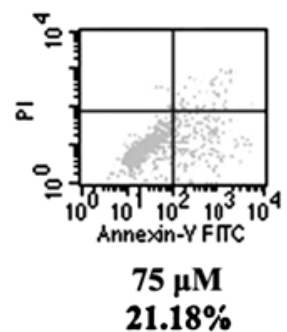

B

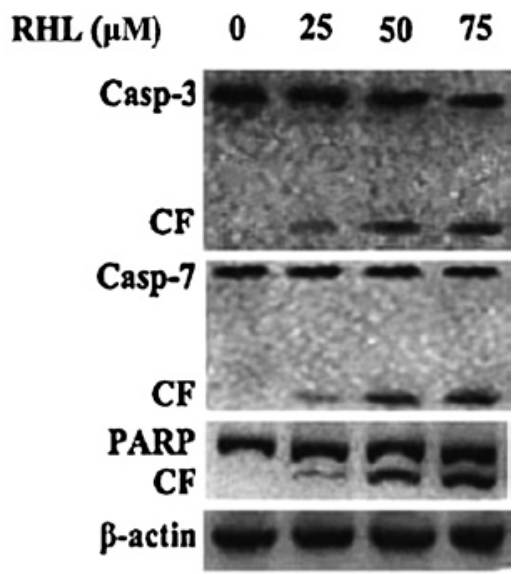

Figure 2. Apoptosis induction by RHL in Hela cells associated with the cleavage of caspase-3/7 and PARP. Cells were treated with RHL for $48 \mathrm{~h}$ at the indicated concentrations and (A) were harvested and labeled with a combination of Annexin V-FITC and propidium iodide. The percentage of early apoptotic cells (lower right quadrant) is shown. (B) The cells were then lysed and subjected to Western blotting using caspase-3/7, PARP and $\beta$-actin antibodies. One representative of three different experiments is shown. Casp, caspase; CF, cleaved fragment.

suggest that RHL inhibits the growth of cervical cancer cells by modulating the activation of various MAPKs.

Effects of mitogen-activated protein kinase on growth inhibition induced by rhein lysinate in HeLa cells. To further examine the role of various MAPKs in the inhibition of cervical cancer cell growth, pharmacological inhibitors of MAPKs (the JNK inhibitor SP600125, the p38 MAPK inhibitor SB203580 and the MEK inhibitor U0126) were used in combination with RHL.

The specificity of each inhibitor for various kinases on RHL-treated cells was assessed. Inhibitors were added and incubated for $1 \mathrm{~h}$, followed by RHL treatment $(75 \mu \mathrm{M})$ for 48 h. As shown in Fig. 4A, the specificity of each inhibitor was confirmed. Moreover, the cleavage of caspase-3/7 and PARP triggered by RHL was blocked by SP600125 and SB203580, whereas the cleavage of caspase-3/7 and PARP triggered by RHL was enhanced by U0126. As shown in Fig. 4B, in the absence of RHL, the inhibitors of JNK, p38 MAPK and MEK
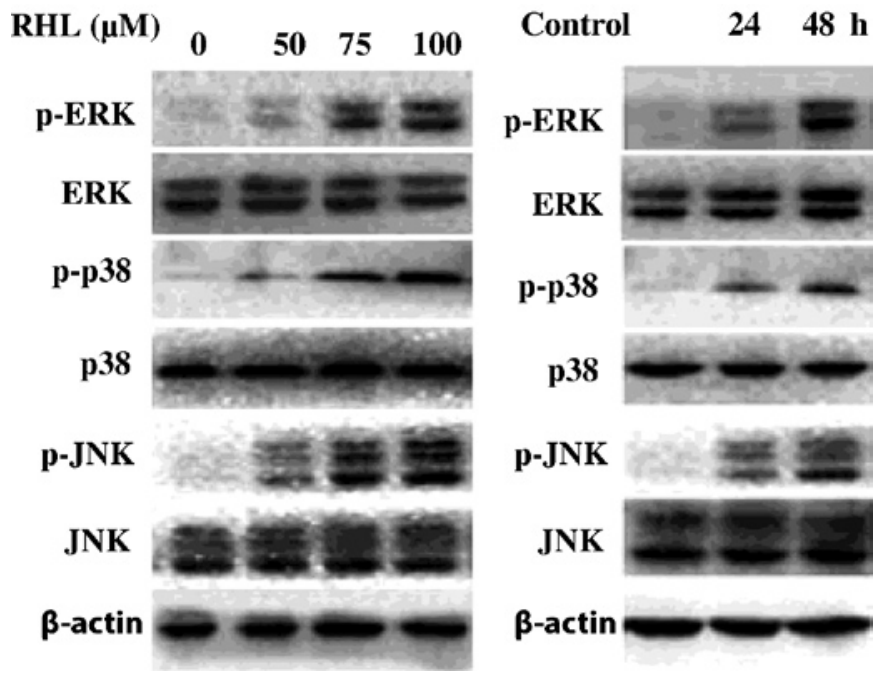

Figure 3. Effect of RHL on MAPKs in Hela cells. (A) The cells were treated with RHL for $48 \mathrm{~h}$ at the indicated concentrations and then harvested, lysed and subjected to Western blotting using p-ERK, ERK, p-p38 MAPK, p38 MAPK, p-JNK, JNK and $\beta$-actin antibodies. (B) The cells were cultured with RHL $(75 \mu \mathrm{M})$ for the indicated times and then harvested, lysed and subjected to Western blotting using the above-mentioned antibodies. Data are representative of three independent experiments.

alone did not significantly alter proliferation in the HeLa cells. When the HeLa cells were treated with RHL in the presence of three inhibitors, respectively, the situation was altered. MTT results showed that inhibition of the p38 MAPK activity with SB203580 and inhibition of the JNK activity with SP600125 rescued RHL-mediated cell growth inhibition in HeLa cells, whereas the inhibition of ERK activity with U0126 enhanced RHL-induced growth inhibition. These results suggest that JNK and p38 MAPK play a partial role in mediating HeLa cell apoptosis triggered by RHL.

\section{Discussion}

Cervical cancer frequently occurs in women in developing countries, and it is the second most frequent cause of cancerrelated death in women worldwide (1). The survival rate after standard treatment for cervical cancer is high. However, recurrence and subsequent resistance to chemoradiation therapy remain a major health issue and the mechanisms involved have yet to be elucidated. Novel agents are required to offer long-term disease control or a potential cure.

Rhein is one of the major bioactive constituents of the rhizome of rhubarb $(2,3)$, and inhibits the proliferation of various human cancer cells (4-8). Our previous study showed that RHL exhibits anti-cancer activity in breast and ovarian cancer, and hepatocellular carcinoma in vivo and in vitro (9-11). In the present study, RHL was shown to inhibit the growth of human cervical cancer cells. The $\mathrm{IC}_{50}$ value of RHL for HeLa cells was $80 \mu \mathrm{M}$. HeLa cells treated with RHL showed an increasing apoptotic ratio in a dose-dependent manner. This increase is related to certain molecular changes involving the activation of MAPKs, cleavage of caspase-3/7 and PARP. It is well known that MAPKs are a family of proteins that transduce signals from the cell membrane to the nucleus in response to a wide range of stimuli. Moreover, 

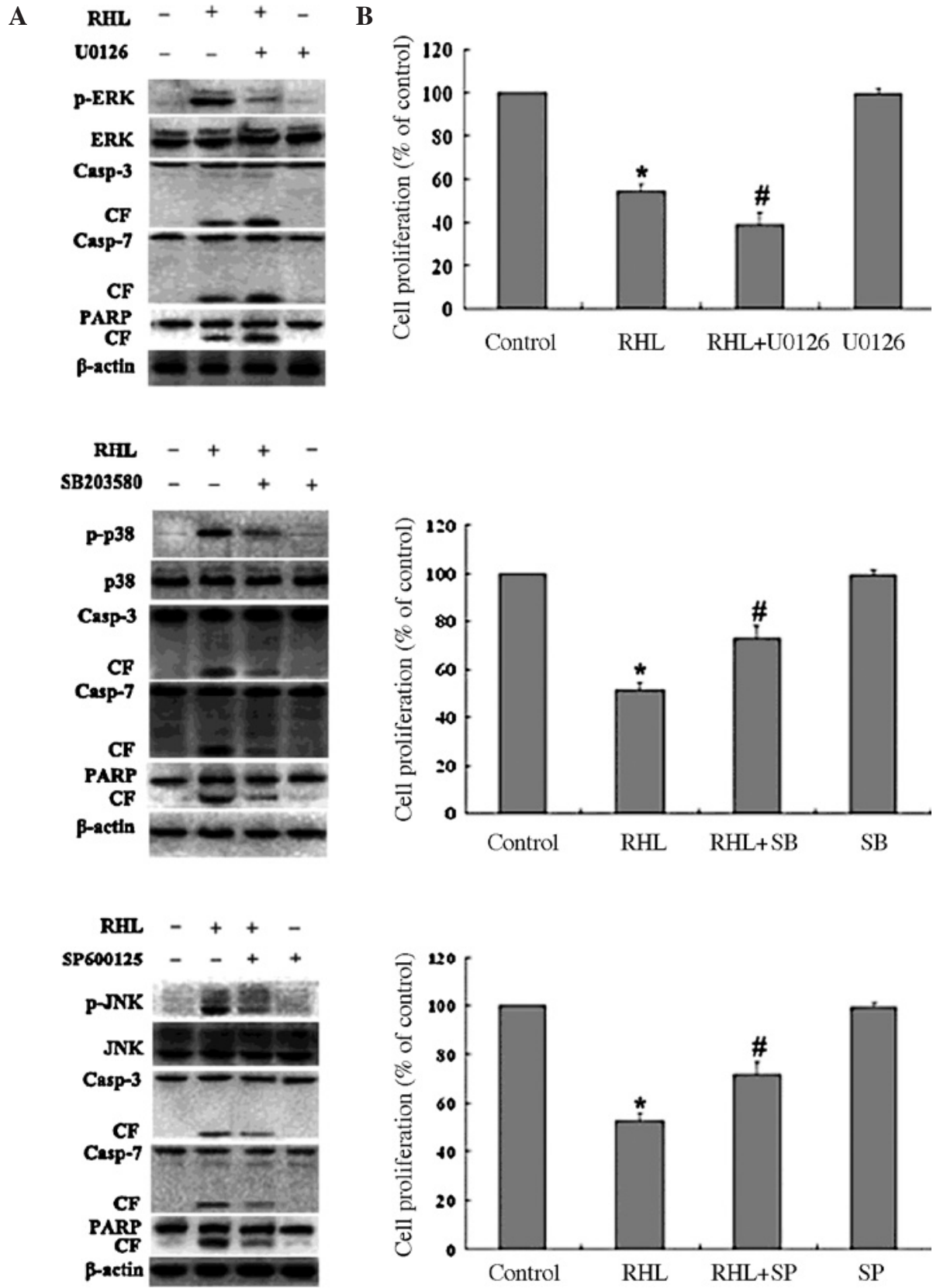

Figure 4. Effect of the MAPK inhibitors on RHL-induced cell growth inhibition. The HeLa cells were pre-treated with inhibitors of MEK (U0126, $10 \mu \mathrm{M}$ ), p38 (SB203580, $10 \mu \mathrm{M}$ ) and JNK (SP600125, $10 \mu \mathrm{M}$ ) for $1 \mathrm{~h}$, followed by RHL (75 $\mu \mathrm{M})$ for an additional $48 \mathrm{~h}$. (A) The specificity of each inhibitor and the level of cleaved caspase-3/7 and PARP were determined by Western blotting. (B) Cell viability was determined with MTT assay in triplicate from three independent experiments. Data are presented as means $\pm \mathrm{SD}$. ${ }^{*} \mathrm{P}<0.05$, control vs. RHL; ${ }^{*} \mathrm{P}<0.05$, RHL vs. inhibitor plus RHL.

MAPKs regulate vital biological functions, including gene expression, mitosis, differentiation, apoptosis, proliferation and motility $(12,13)$. There are three major groups of MAPKs, i.e., JNK, p38 MAPK and ERK. The involvement of JNK in response to stress stimuli, cytokines and anti-cancer agents was previously documented (14-17). In our previous study, JNK played a crucial role in growth inhibition induced by lidamycin in human multiple myeloma cells $(18,19)$. In the present study, RHL triggered JNK phosphorylation in a dosedependent manner in HeLa cells. The results showed that the JNK inhibitor SP600125 markedly attenuated RHL-induced growth inhibition in HeLa cells. The results suggest that the activation of JNK plays a crucial role in growth inhibition induced by RHL in HeLa cells. p38 MAPK has been shown to mediate both proapoptotic/ growth inhibitory and antiapoptotic/progrowth signals in various systems, depending on the stimulus and cell type involved (13,20-22). Since RHL triggered the phosphorylation of p38 MAPK, we examined the role of p38 MAPK activation in HeLa cells to RHL treatment. The results showed that the p38 MAPK inhibitor SB203580 markedly rescued RHL-induced growth inhibition in HeLa cells.

The results suggest that the activation of p38 MAPK also plays a partial role in mediating HeLa cell growth inhibition triggered by RHL in HeLa cells.

The decrease of HeLa cell growth inhibition by the combination of RHL with SP600125 or SB203580 was accompanied by the inactivation of caspase-3/7 and PARP, suggesting that 
the sustained activation of JNK and p38 MAPK plays a critical role in RHL-induced apoptosis in HeLa cells.

The activation of ERK was crucial in mediating proliferation in cancer cells $(19,20,23-25)$. In the present study, RHL triggered significant up-regulation of ERK phosphorylation. We hypothesized that this up-regulation is a compensatory positive feedback response to maintain HeLa cell survival. Inhibiting MEK/ERK signaling would therefore enhance RHL-induced growth inhibition in HeLa cells. To inhibit up-regulation of MEK/ERK activity triggered by RHL, we utilized the MEK1/2 inhibitor, U0126, which inhibits ERK activity. Growth inhibition triggered by RHL was synergistically augmented in the presence of U0126. The results suggest the potential of MEK/ERK inhibition to enhance sensitivity to RHL and to overcome RHL resistance, thereby improving the therapeutic efficacy of RHL in HeLa cells. The increase of HeLa cell growth inhibition by the combination of RHL with U0126 was accompanied by the enhancement of the activation of caspase-3/7 and PARP, suggesting that ERK is involved in regulating cell survival. The finding that RHL inhibited ERK activation was shown in another study using human mammary epithelial MCF cells (9). However, in our study, the activation of ERK was increased following RHL treatment in HeLa cells. These findings indicate that ERK is activated or inhibited by RHL in a cell type-specific manner.

RHL activates the three members of MAPKs, thus modulating the balance between the survival and death signaling cascades, thereby inducing growth inhibition. Taken together, these results suggest that RHL inhibits cell growth via the activation of p38 MAPK and JNK. The results provide the rationale for clinical trials of RHL aimed at improving patient outcome in cervical cancer.

\section{Acknowledgements}

We would like to thank Professor Yong-Shu Zhen (Department of Oncology, Institute of Medicinal Biotechnology, Chinese Academy of Medical Science, Beijing, China) for kindly providing us with the rights to use Patent No. 200810089025.8.

\section{References}

1. Parkin DM, Bray F, Ferlay J and Pisani P: Global cancer statistics, 2002. CA Cancer J Clin 55: 74-108, 2005.

2. Kuo PL, Hsu YL, Ng LT and Lin CC: Rhein inhibits the growth and induces the apoptosis of Hep G2 cells. Planta Med 70: 12-16, 2004.

3. Huang Q, Lu G, Shen HM, Chung MC and Ong CN: Anti-cancer properties of anthraquinones from rhubarb. Med Res Rev 27: 609-630, 2007

4. Lai WW, Yang JS, Lai KC, et al: Rhein induced apoptosis through the endoplasmic reticulum stress, caspase-and mitochondriadependent pathways in SCC-4 human tongue squamous cancer cells. In Vivo 23: 309-316, 2009.

5. Ip SW, Weng YS, Lin SY, et al: The role of $\mathrm{Ca}^{2+}$ on rhein-induced apoptosis in human cervical cancer Ca Ski cells. Anticancer Res 27: 379-389, 2007
6. Lin ML, Chen SS, Lu YC, et al: Rhein induces apoptosis through induction of endoplasmic reticulum stress and $\mathrm{Ca}^{2+}$-dependent mitochondrial death pathway in human nasopharyngeal carcinoma cells. Anticancer Res 27: 3313-3322, 2007.

7. Cichewicz RH, Zhang Y, Seeram NP and Nair MG: Inhibition of human tumor cell proliferation by novel anthraquinones from daylilies. Life Sci 74: 1791-1799, 2004.

8. Floridi A, Gentile PF, Bruno T, et al: Cytotoxic effect of the association of BCNU with rhein or lonidamine on a human glioma cell line. Anticancer Res 11: 789-792, 1991.

9. Lin YJ and Zhen YS: Rhein lysinate suppresses the growth of breast cancer cells and potentiates the inhibitory effect of Taxol in athymic mice. Anticancer Drugs 20: 65-72, 2009.

10. Lin YJ, Zhen YZ, Shang BY and Zhen YS: Rhein lysinate suppresses the growth of tumor cells and increases the antitumor activity of Taxol in mice. Am J Chin Med 37: 923-931, 2009.

11. Lin YJ, Huang YH, Zhen YZ, Liu XJ and Zhen YS: Rhein lysinate induces apoptosis in breast cancer SK-Br-3 cells by inhibiting HER-2 signal pathway. Yao Xue Xue Bao 43: 1099-1105, 2008.

12. Schaeffer HJ and Weber MJ: Mitogen-activated protein kinases: specific messages from ubiquitous messengers. Mol Cell Biol 19: 2435-2444, 1999.

13. Wen J, Cheng HY, Feng Y, et al: p38 MAPK inhibition enhancing ATO-induced cytotoxicity against multiple myeloma cells. Br J Haematol 140: 169-180, 2008.

14. Shtil AA, Mandlekar S, Yu R, et al: Differential regulation of mitogen-activated protein kinases by microtubule-binding agents in human breast cancer cells. Oncogene 18: 377-384, 1999.

15. Kyriakis JM and Avruch J: Mammalian mitogen-activated protein kinase signal transduction pathways activated by stress and inflammation. Physiol Rev 81: 807-869, 2001.

16. Wang TH, Wang HS, Ichijo $\mathrm{H}$, et al: Microtubule-interfering agents activate c-Jun $\mathrm{N}$-terminal kinase/stress-activated protein kinase through both Ras and apoptosis signal-regulating kinase pathway. J Biol Chem 273: 4928-4936, 1998.

17. Stone AA and Chambers TC: Microtubule inhibitors elicit differential effects on MAP kinase (JNK, ERK, and p38) signaling pathways in human KB-3 carcinoma cells. Exp Cell Res 254: $110-119,2000$.

18. Zhen YZ, Lin YJ, Li Y and Zhen YS: Lidamycin shows highly potent cytotoxic to myeloma cells and inhibits tumor growth in mice. Acta Pharmacol Sin 30: 1025-1032, 2009.

19. Zhen YZ, Lin YJ, Shang BY and Zhen YS: Enediyne lidamycin induces apoptosis in human multiple myeloma cells through activation of p38 mitogen-activated protein kinase and c-Jun NH2-terminal kinase. Int J Hematol 90: 44-51, 2009.

20. Hideshima T, Catley L, Yasui $\mathrm{H}$, et al: Perifosine, an oral bioactive novel alkylphospholipid, inhibits Akt and induces in vitro and in vivo cytotoxicity in human multiple myeloma cells. Blood 107: 4053-4062, 2006.

21. Park WH, Seol JG, Kim ES, et al: Induction of apoptosis by vitamin D3 analogue EB1089 in NCI-H929 myeloma cells via activation of caspase 3 and p38 MAP kinase. Br J Haematol 109: 576-583, 2000.

22. Shimizu T, Nakazato T, Xian MJ, Sagawa M, Ikeda Y and Kizaki M: Resveratrol induces apoptosis of human malignant B cells by activation of caspase- 3 and p38 MAP kinase pathways. Biochem Pharmacol 71: 742-750, 2006.

23. Pei XY, Dai Y, Tenorio S, et al: MEK1/2 inhibitors potentiate UCN-01 lethality in human multiple myeloma cells through a Bim-dependent mechanism. Blood 110: 2092-2101, 2007.

24. Lunghi P, Giuliani N, Mazzera L, et al: Targeting MEK/MAPK signal transduction module potentiates ATO-induced apoptosis in multiple myeloma cells through multiple signaling pathways. Blood 112: 2450-2462, 2008.

25. Ozaki K, Kosugi M, Baba N, et al: Blockade of the ERK or PI3K-Akt signaling pathway enhances the cytotoxicity of histone deacetylase inhibitors in tumor cells resistant to gefitinib or imatinib. Biochem Biophys Res Commun 391: 1610-1615, 2010. 